\title{
Plasma soluble urokinase plasminogen activator receptor (suPAR) levels in systemic lupus erythematosus
}

\author{
Gergely Toldi, Balázs Szalay, Gabriella Bekő, Márta Bocskai, Magdolna Deák, \\ László Kovács, Barna Vásárhelyi \& Attila Balog
}

To cite this article: Gergely Toldi, Balázs Szalay, Gabriella Bekő, Márta Bocskai, Magdolna Deák, László Kovács, Barna Vásárhelyi \& Attila Balog (2012) Plasma soluble urokinase plasminogen activator receptor (suPAR) levels in systemic lupus erythematosus, Biomarkers, 17:8, 758-763, DOI: 10.3109/1354750X.2012.728623

To link to this article: http://dx.doi.org/10.3109/1354750X.2012.728623

Published online: 04 Oct 2012.

Submit your article to this journal $₫$

Џll Article views: 129

Q View related articles $\sqsubset$ 


\title{
Plasma soluble urokinase plasminogen activator receptor (suPAR) levels in systemic lupus erythematosus
}

\author{
Gergely Toldi ${ }^{1}$, Balázs Szalay², Gabriella Bekő², Márta Bocskai ${ }^{3}$, Magdolna Deák³, László Kovács ${ }^{3}$, \\ Barna Vásárhelyi ${ }^{2}$, and Attila Balog ${ }^{3}$ \\ ${ }^{1}$ First Department of Pediatrics, Semmelweis University, Budapest, Hungary, ${ }^{2}$ Department of Laboratory Medicine, \\ Semmelweis University, Budapest, Hungary, and ${ }^{3}$ Department of Rheumatology, Albert Szent-Györgyi Health Center, \\ University of Szeged, Szeged, Hungary
}

\begin{abstract}
Objective: Soluble urokinase plasminogen activator receptor (suPAR) is a biomarker of systemic inflammation. We aimed to characterize plasma suPAR levels in SLE patients.

Methods: We measured plasma suPAR, C reactive protein (CRP) and erythrocyte sedimentation rate (ESR) in 89 SLE patients and 29 healthy controls.

Results: SUPAR and ESR values were higher in SLE than in controls, while CRP levels were comparable. ROC analysis of suPAR levels indicated a cut-off value of $5.70 \mathrm{ng} / \mathrm{mL}$ to distinguish patients with high disease activity (SLEDAI >8). Conclusion: suPAR might be an objective marker for identifying SLE patients with active disease.
\end{abstract}

Keywords: CRP, inflammation, SLE, suPAR, vasculitis

\section{Introduction}

Systemic lupus erythematosus (SLE) is one of the mostinvestigated autoimmune disorders, characterized by a systemic inflammatory reaction. It affects nearly all vital organs and tissues and is characterized by a wide spectrum of clinical signs and symptoms.

The diagnosis of SLE is based on thorough clinical assessment of the patient. The disease course is fluctuating, characterized by acute and often severe exacerbations. Several scoring systems have been validated to measure disease activity (Griffiths et al. 2005); however, no objective laboratory marker has been identified that can reliably be used for the detection of ongoing inflammation in correlation with clinical symptoms in SLE.

Nowadays $C$ reactive protein (CRP) is regarded as the gold-standard for the assessment of systemic inflammation. It is used not only in infections, but also in several immune-mediated conditions, such as rheumatoid arthritis (RA). However, in SLE, CRP levels are often not elevated. Previous studies demonstrated a failure of the acute phase CRP response during active lupus despite evident tissue inflammation (Russell et al. 2004). In clinical practice, a significantly elevated erythrocyte sedimentation rate (ESR) with a normal CRP is a strong indicator of SLE. ESR is, however, a rather unspecific marker of inflammation.

It is not completely clear why - in contrast with other rheumatic diseases - SLE is characterized by a low CRP response, in spite of a significant degree of tissue inflammation. Induction of CRP synthesis in hepatocytes is regulated by pro-inflammatory cytokines, most importantly by IL-6, IL-1b and TNF-a. Liou demonstrated that serum levels of these cytokines in patients with untreated lupus were higher than in controls, but still lower compared to RA. Furthermore, cytokine and CRP levels were not proportionally correlated, indicating that these cytokines might insufficiently induce CRP synthesis in SLE (Liou 2001). 
Over the recent years, soluble urokinase plasminogen activator receptor (suPAR) has been described as a valuable indicator of the activation state of the immune system. Urokinase-type plasminogen activator receptor (UPAR) is expressed on various cell types, including immune, smooth muscle and endothelial cells (Danø et al. 1994; Behrendt \& Stephens 1998). This membrane protein may be cleaved from the cell surface, thus forming a free soluble receptor, suPAR (Stephens et al. 1997). suPAR is detectable in low, but practically constant concentrations in plasma of healthy individuals (Rønne et al. 1995; Stephens et al. 1997). However, an inflammatory response leads to elevated plasma suPAR levels, as reported in conditions such as infectious (Ostergaard etal.2004; Ostrowski etal. 2005), autoimmune (Balabanov et al. 2001), neoplastic (Sier et al. 1998) and pregnancyrelated (Toldi et al. 2011) diseases. Importantly, higher levels of suPAR were proportional to a worse prognosis in the above conditions (Sier et al. 1998; Ostergaard et al. 2004; Ostrowski et al. 2005).

suPAR's high stability in plasma samples makes it an ideal candidate as a potential clinical marker. In contrast to CRP, suPAR levels are stable throughout the day in healthy individuals, independently of whether the subject is fasting or not (Sier et al. 1999). Even repeated freeze-thaw procedures of plasma samples did not affect suPAR concentrations (Riisbro et al. 2001).

In this study, we aimed to determine plasma suPAR levels in SLE patients compared to healthy individuals in order to reveal if suPAR could serve as an activity biomarker in this disease, and differentiate between clinical SLE subgroups.

\section{Methods}

\section{Study participants}

Eighty-nine SLE patients with various stages of disease duration and activity and 29 healthy individuals were enrolled in this study. SLE patients were diagnosed and classified according to the updated American College of Rheumatology (ACR97) criteria (Hochberg 1997). Clinical parameters of study participants are summarized in Table 1. All SLE patients were diagnosed at least two years before sampling. The median of SLE duration was 8 years, and the median of Systemic Lupus Erythematosus Disease Activity Index (SLEDAI) score was 2, corresponding to moderate disease activity (Griffiths et al. 2005). SLEDAI scores were calculated at the time of sampling. Patients were treated with the following medications: methylprednisolone (73\%), chloroquine (45\%), azathioprine (21\%), cyclophosphamide (11\%), methotrexate (11\%), cyclosporin (4\%), and mycophenolate mofetil (2\%). Healthy controls were volunteer blood donors and had a negative history of rheumatic symptoms and negative status upon detailed physical and routine laboratory examination. Written informed consent was obtained from all subjects, and our study was reviewed and approved by an independent ethical committee of the
Table 1. Clinical characteristics of healthy individuals and systemic lupus erythematosus (SLE) patients.

\begin{tabular}{lccc}
\hline & $\begin{array}{c}\text { Healthy } \\
\text { individuals } \\
(\mathrm{n}=29)\end{array}$ & $\begin{array}{c}\text { SLE patients } \\
(\mathrm{n}=89)\end{array}$ & $p$ value \\
\hline Characteristics & $55[46-69]$ & $44[34-59]$ & 0.25 \\
Age (years) & $10 / 18$ & $10 / 79$ & - \\
Gender (male/female) & - & $8[3-13]$ & - \\
SLE duration (years) & - & $2[0-5]$ & - \\
SLEDAI & - & $26[12-67]$ & - \\
Anti-DNA (IU/mL) & - & $107[76-130]$ & - \\
C3 (mg/dL) & - & $17[12-23]$ & - \\
C4 (mg/dL) & $2.80[2.06-3.42]$ & $4.58^{\mathrm{a}}[3.72-6.30]$ & 0.0001 \\
suPAR (ng/mL) & $2.70[\mathrm{BLD}-4.15]$ & $3.90[\mathrm{BLD}-9.55]$ & 0.14 \\
$\mathrm{CRP}(\mathrm{mg} / \mathrm{L})$ & $10[7-14]$ & $28^{\mathrm{a}}[17-50]$ & 0.0001 \\
ESR $(\mathrm{mm} / \mathrm{h})$ &
\end{tabular}

Data are expressed as median [interquartile range].

${ }^{\mathrm{a}} p<0.01$ vs. healthy individuals.

$\mathrm{BLD}$, below the level of detection, CRP, C reactive protein, ESR, erythrocyte sedimentation rate, SLE, systemic lupus erythematosus, SLEDAI, systemic lupus erythematosus disease activity index, suPAR, soluble urokinase plasminogen activator receptor.

institution. Laboratory studies and interpretations were performed on coded samples lacking personal and diagnostic identifiers. The study was adhered to the tenets of the most recent revision of the Declaration of Helsinki.

\section{Laboratory procedures}

Plasma was isolated from EDTA anticoagulated fasting blood samples and stored at $-80^{\circ} \mathrm{C}$ until measurement. Plasma suPAR concentrations were measured with the suPARnostic Flex ELISA assay (ViroGates A/S, Birkerød, Denmark). hs-CRP levels were measured using a Roche Hitachi 912 instrument with Roche Tina-quant CRP immuno-turbidimetric assay (Roche Diagnostics $\mathrm{GmbH}$, Mannheim, Germany). For the determination of erythrocyte sedimentation rate (ESR), the Westergren method was performed according to ICSH specifications (International Council for Standardization in Haematology 1993) on undiluted EDTA anticoagulated blood samples using glass pipettes (Greiner Bio-One, Kremsmuenster, Austria). During sedimentation, the pipettes were mounted vertically on appropriate supporting racks and kept at room temperature, which never exceeded $25^{\circ} \mathrm{C}$.

\section{Statistics}

Data are expressed as median [interquartile range]. CRP values below the level of detection $(1 \mathrm{mg} / \mathrm{L})$ were regarded as $1 \mathrm{mg} / \mathrm{L}$. Comparisons between healthy individuals and SLE patients were made with MannWhitney tests. In case of comparisons between healthy controls and SLE patient subgroups, the Kruskal-Wallis test was performed. To study the independent effect of the investigated variables, multiple regression analysis was used. AUC values of receiver operating characteristics (ROC) curves were calculated using standard methods and data are presented as AUC ROC (95\% CI). 
$p$ values $<0.05$ were considered significant. Statistics were calculated using the SPSS software (version 20.0, SPSS, Inc. Chicago, IL, USA).

\section{Results}

suPAR and ESR values were higher in SLE patients than in controls, while CRP levels were comparable (Table 1, Figure 1).

We performed further analyses of suPAR levels based on several subgroups of SLE patients. We investigated patients with or without the following factors occurring in their history: arthritis (89\%), photosensitivity (67\%), butterfly rash $(20 \%)$, other skin lesion $(42 \%)$, serositis $(37 \%)$, pericarditis $(30 \%)$, pleuritis $(38 \%)$, nephritis (46\%), Raynaud's phenomenon (56\%), vasculitis (28\%), thromboembolism (20\%), adenomegaly (36\%), splenomegaly (11\%), secondary Sjögren's syndrome (20\%), hemolytic anemia (hematocrit $<0.35,13 \%$ ), nonhemolytic anemia (hematocrit $<0.35,65 \%$ ), leukopenia (leukocyte count $<4000 / \mathrm{mm}^{3}, 62 \%$ ), lymphopenia (lymphocyte count $<1500 / \mathrm{mm}^{3}, 61 \%$ ), thrombocytopenia (thrombocyte count $<100000 / \mathrm{mm}^{3}, 19 \%$ ), anti-DNA antibody positivity (81\%), anti-SSA antibody positivity (52\%), anti-SSB antibody positivity (35\%), anti-Sm antibody positivity (26\%), anti-RNP antibody positivity (22\%), anti-CL antibody positivity (42\%), anti-beta2GPI antibody positivity (29\%), lupus anticoagulant positivity (activated partial thromboplastin time $>40 \mathrm{~s}, 30 \%$ ), low C3 complement level (78\%), low C4 complement level (57\%) and Coombs positivity (17\%).

suPAR levels of patients with vasculitis in their history (28\%) was higher than that of patients with no vasculitis (5.84 [4.12-7.01] vs. 4.21 [3.57-5.47] ng/mL, $p=0.04$ ). In cases of all other comparisons, no differences were detected between lupus patient subgroups in suPAR levels.

When SLE patients were divided into subgroups based on CRP $(\leq 5 \mathrm{mg} / \mathrm{L}$ and $>5 \mathrm{mg} / \mathrm{L})$ and ESR values ( $\leq 20 \mathrm{~mm} / \mathrm{h}$ and $>20 \mathrm{~mm} / \mathrm{h}$ ), no difference was detected in suPAR levels between the subgroups (Table 2).

suPAR, CRP and ESR values were also analyzed in SLE patient subgroups based on SLEDAI scores (Table 3,
Figure 2). Patients with a SLEDAI score of 0 were considered to be in remission, a SLEDAI score between 1 and 8 was regarded as moderate disease activity, and a SLEDAI score above 8 was regarded as high disease activity. In case of CRP and ESR values, no difference was detected between SLE patients grouped according to SLEDAI scores. However, interestingly, suPAR levels in patients with high disease activity were higher than in patients with moderate disease activity or patients in remission.

ROC analysis of suPAR values in healthy individuals and SLE patients yielded an AUC of 0.85 (95\% CI: $0.77-0.93, p=0.0001$, Figure 3 ). The cut-off value of suPAR to discriminate between healthy individuals and SLE patients was $3.54 \mathrm{ng} / \mathrm{mL}$ (sensitivity\% (95\% CI): 82.02 (72.45-89.63), specificity\% (95\% CI): 79.31 (60.2892.01)). ROC analysis of CRP levels in healthy individuals and SLE patients did not present a statistically significant AUC value. ROC analysis of ESR values in healthy individuals and SLE patients provided an AUC of 0.87 (95\% CI: $0.80-0.94, p=0.0001$ ).

ROC analysis of suPAR values in SLE patients according to SLEDAI scores yielded an AUC of 0.68 (95\% CI: $0.50-0.86, p=0.04$, Figure 3 ). The cut-off value of suPAR to discriminate between patients with high disease activity (SLEDAI > 8) and those with moderate disease activity or in remission (SLEDAI $\leq 8$ ) was $5.70 \mathrm{ng} / \mathrm{mL}$ (sensitivity\% (95\% CI): 61.54 (31.58-86.14), specificity\% (95\% CI): 78.72 (64.34-89.30)). ROC analysis of CRP and ESR values in SLE patients according to SLEDAI scores did not present a statistically significant AUC value.

\section{Discussion}

We have demonstrated that plasma levels of suPAR, a novel marker of systemic inflammation are elevated in lupus patients compared to healthy individuals (cut-off value: $3.54 \mathrm{ng} / \mathrm{mL}$ ). ESR values were also higher in the SLE group, while CRP levels were comparable in the healthy and diseased groups. Of note, the SLE group had a higher female to male ratio compared to the control group, in line with unequal gender prevalence of the disease. The effect of gender on our results cannot be excluded, since according to earlier data, slightly higher
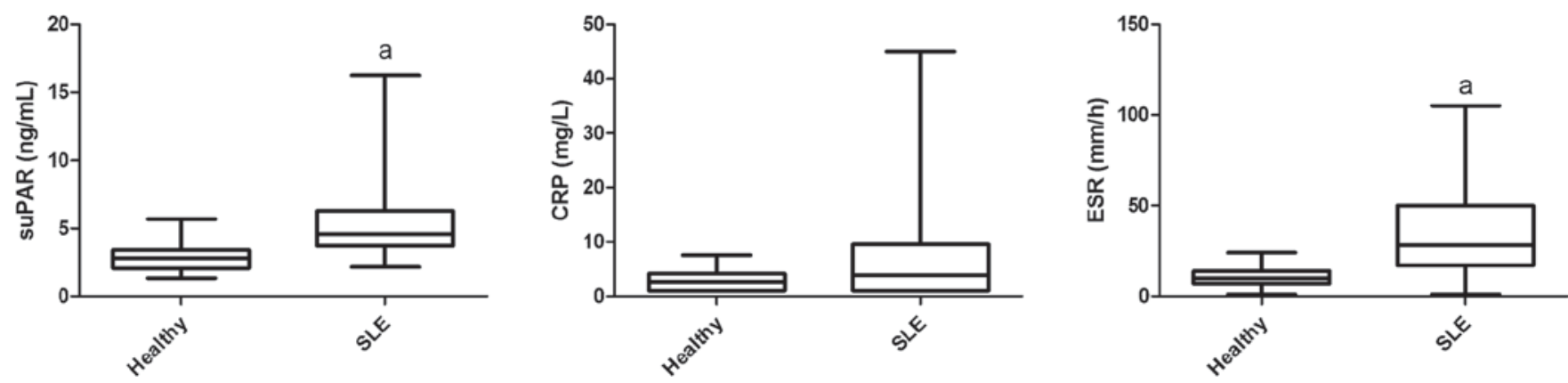

Figure 1. Comparison of soluble urokinase plasminogen activator receptor (suPAR) plasma levels, C reactive protein (CRP) plasma levels and erythrocyte sedimentation rate (ESR) in healthy individuals and systemic lupus erythematosus (SLE) patients. Horizontal line: median; box: interquartile range; whisker: range. ${ }^{\mathrm{a}} p<0.05$ vs. healthy individuals. 
suPAR concentrations were observed in serum from females compared to males (Stephens et al. 1997).

When CRP levels rise above $60 \mathrm{mg} / \mathrm{L}$ in febrile SLE patients, it is highly sensitive for infection. In contrast, in SLE without infection, CRP levels are only moderately raised even in patients with very active disease (ter Borg etal.1990). Over the recentyears, at least three contributing

Table 2. Soluble urokinase plasminogen activator receptor (suPAR) levels in subgroups of systemic lupus erythematosus (SLE) patients according to inflammatory parameters.

\begin{tabular}{lc}
\hline Study group & suPAR $(\mathrm{ng} / \mathrm{mL})$ \\
\hline Healthy individuals & $2.80[2.06-3.42]$ \\
SLE patients according to CRP values & \\
$\quad \leq 5 \mathrm{mg} / \mathrm{L}(58.4 \%)$ & $4.09^{\mathrm{a}}[3.46-5.94]$ \\
$\quad>5 \mathrm{mg} / \mathrm{L}(41.6 \%)$ & $5.00^{\mathrm{a}}[4.12-7.19]$ \\
SLE patients according to ESR values & \\
$\quad \leq 20 \mathrm{~mm} / \mathrm{h}(38.2 \%)$ & $4.02^{\mathrm{a}}[3.01-5.51]$ \\
$>20 \mathrm{~mm} / \mathrm{h}(61.8 \%)$ & $4.83^{\mathrm{a}}[3.94-6.92]$ \\
\hline
\end{tabular}

Data are expressed as median [interquartile range].

${ }^{\mathrm{a}} p<0.05$ vs. healthy individuals.

CRP, C reactive protein; ESR, erythrocyte sedimentation rate; SLE, systemic lupus erythematosus; suPAR, soluble urokinase plasminogen activator receptor.

Table 3. Inflammatory parameters in systemic lupus erythematosus (SLE) patients according to Systemic Lupus Erythematosus Disease Activity Index (SLEDAI).

\begin{tabular}{|c|c|c|c|}
\hline Study group & suPAR (ng/mL) & $\mathrm{CRP}(\mathrm{mg} / \mathrm{L})$ & $\operatorname{ESR}(\mathrm{mm} / \mathrm{h})$ \\
\hline $\begin{array}{l}\text { Healthy } \\
\text { individuals }\end{array}$ & $2.80[2.06-3.42]$ & 2.70 [BLD-4.15] & $10[7-14]$ \\
\hline \multicolumn{4}{|c|}{ SLE patients according to SLEDAI score } \\
\hline $0(32.6 \%)$ & $4.09^{\mathrm{a}}[3.45-7.53]$ & $4.20[\mathrm{BLD}-8.20]$ & $27^{\mathrm{a}}[15-50]$ \\
\hline $1-8(52.8 \%)$ & $4.58^{\mathrm{a}}[3.75-5.41]$ & 2.20 [BLD-10.10] & $29^{\mathrm{a}}[15-51]$ \\
\hline$>8(14.6 \%)$ & $\begin{array}{c}6.70^{\mathrm{a}, \mathrm{b}, \mathrm{c}} \\
{[4.17-8.71]}\end{array}$ & $\begin{array}{c}6.70^{\mathrm{a}} \\
{[3.14-18.60]}\end{array}$ & $\begin{array}{c}36^{\mathrm{a}} \\
{[21-47]}\end{array}$ \\
\hline
\end{tabular}

Data are expressed as median [interquartile range].

${ }^{\mathrm{a}} p<0.05$ vs. healthy individuals.

${ }^{\mathrm{b}} p<0.05$ vs. SLE patients according to SLEDAI score 0 .

${ }^{\mathrm{c}} p<0.05$ vs. SLE patients according to SLEDAI score 1-8.

$\mathrm{BLD}$, below the level of detection; $\mathrm{CRP}, \mathrm{C}$ reactive protein; $\mathrm{ESR}$, erythrocyte sedimentation rate; SLE, systemic lupus erythematosus; SLEDAI, systemic lupus erythematosus disease activity index; suPAR, soluble urokinase plasminogen activator receptor. factors have been proposed to understand why CRP levels are lower in SLE compared to other inflammatory autoimmune disorders, such as RA (de Carvalho et al. 2007). These include the different responsiveness of monocytes producing CRP-inducing cytokines in SLE (Liou 2003), and the role of human CRP gene polymorphisms. Two of these, CRP 2 and CRP 4, were associated with lower CRP levels. Moreover, the CRP 4 allele was also linked with the future development of SLE (Russell et al. 2004). Third, among several autoimmune disorders, Sjöwall et al. (2002) investigated SLE patients for autoantibodies to CRP. Serum anti-CRP autoantibodies were found in almost half of SLE patients, whereas no anti-CRP antibodies were detected in any of the samples from patients with RA, Crohn's disease or ulcerative colitis. Szyper-Kravitz and Shoenfeld (2006) have recently suggested that antiCRP autoantibodies, by binding or inactivating CRP, a molecule involved in the clearance of apoptotic cells, may serve as the promoter of autoimmunity in SLE.

Our results suggest that suPAR levels are higher in SLE patients with vasculitis in their history than in patients without vasculitis. Experimentally, lower expression of UPAR on the endothelial cell surface is associated with up to $60 \%$ reduced cellular adhesion and extravasation of inflammatory cells into the intima of vessel walls (May et al. 2000). A study by May et al. (2000) elucidated a novel activation pathway of beta(2) integrin-dependent cellto-cell adhesion during inflammation that requires UPAR as an activation transducer. In their experiments, adhesion was reconstituted when soluble recombinant uPAR was allowed to reassociate with the cells. Although we did not aim to investigate endothelial uPAR expression in our study, one might hypothesize that inflammatory cell adhesion to endothelial cells leading to extravasation into the intima is promoted by higher suPAR levels leading to the development of vasculitis in patients with elevated plasma suPAR levels. suPAR might promote cell adhesion by binding to very late antigen-4 (VLA-4) on inflammatory cells as a ligand, promoting their extravasation via the activation of other molecules regulating cellular adhesion and migration (Tarui et al. 2001).
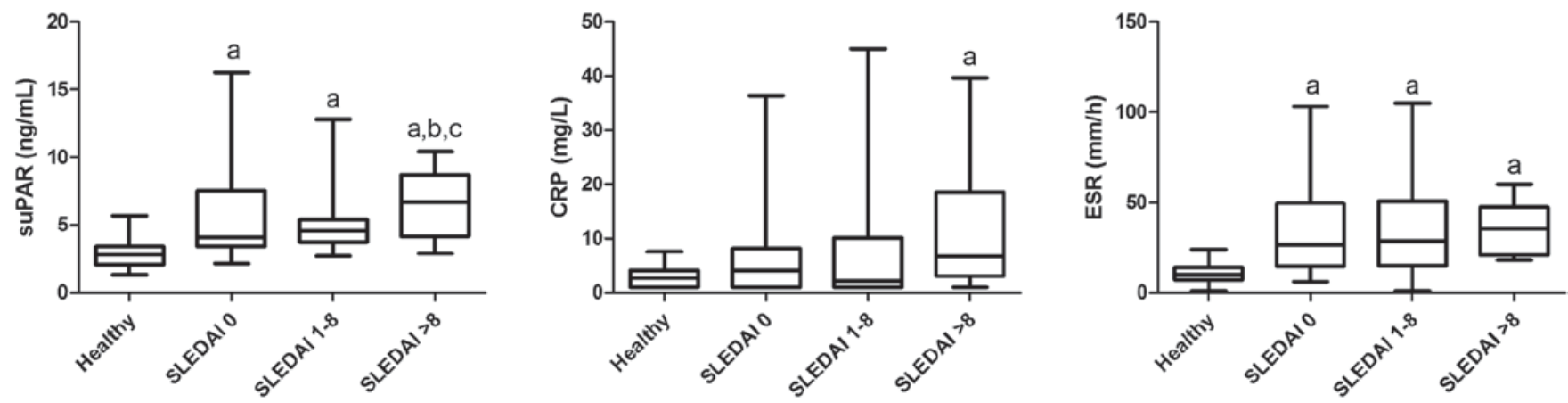

Figure 2. Comparison of soluble urokinase plasminogen activator receptor (suPAR) plasma levels, C reactive protein (CRP) plasma levels and erythrocyte sedimentation rate (ESR) in healthy individuals and systemic lupus erythematosus (SLE) patients grouped according to Systemic Lupus Erythematosus Disease Activity Index (SLEDAI) scores. Patients with a SLEDAI score of 0 were considered to be in remission, a SLEDAI score between 1 and 8 was regarded as moderate disease activity, and a SLEDAI score above 8 was regarded as high disease activity. Horizontal line: median, box: interquartile range, whisker: range. ${ }^{\mathrm{a}} p<0.05$ vs. healthy individuals, ${ }^{\mathrm{b}} p<0.05$ vs. SLE patients with SLEDAI 0 , ${ }^{\mathrm{c}} p<0.05$ vs. SLE patients with SLEDAI $1-8$. 
ROC of SuPAR values in healthy individuals and SLE patients

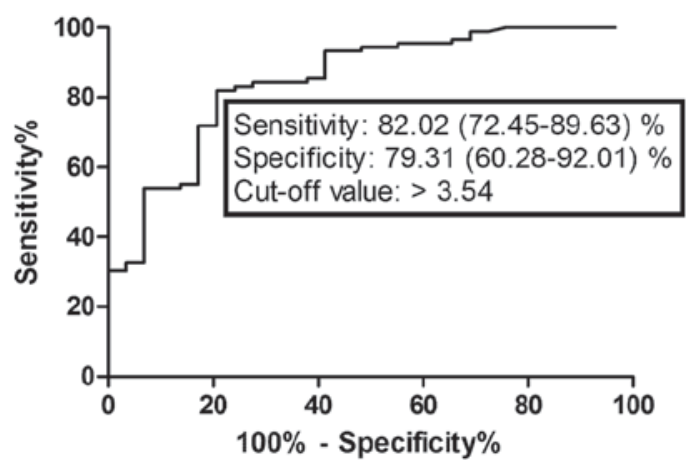

ROC of SUPAR values in SLE patients according to SLEDAl scores

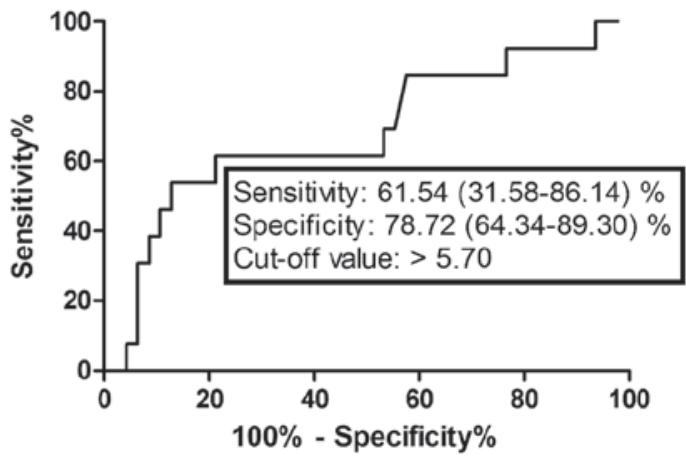

Figure 3. Receiver operator curve (ROC) analysis of soluble urokinase plasminogen activator receptor (suPAR) values in healthy individuals and SLE patients, as well as in SLE patients according to disease activity (Systemic Lupus Erythematosus Disease Activity Index (SLEDAI) scores). Patients with a SLEDAI score $>8$ were regarded as high disease activity, while those with a SLEDAI score $\leq 8$ were regarded as moderate disease activity or in remission. AUC in healthy individuals and SLE patients: 0.85 (95\% CI: 0.77-0.93, $p=0.0001$ ). AUC in SLE patients according to SLEDAI scores: 0.68 (95\% CI: 0.50-0.86, $p=0.04$ ).

Importantly, our results indicate that suPAR, in contrast with CRP and ESR, is a marker that may help to discriminate between patients with high disease activity (SLEDAI $>8$ ) and those with moderate disease activity or in remission (SLEDAI $\leq 8)$. Therefore, with a calculated cut-off value of $5.70 \mathrm{ng} / \mathrm{mL}$, suPAR levels may be considered as an objective marker of highly active SLE. Based on our recent findings, suPAR levels are also elevated in RA patients, and are is associated with the number of swollen joints (Toldi et al. 2012). Therefore, suPAR might be regarded as a general marker of disease activity in patients with autoimmune disease.

\section{Conclusions}

Unlike CRP levels, suPAR values are higher in SLE patients than in healthy controls. The assessment of inflammation is of special importance in the timely detection and treatment of potentially dangerous flares of SLE. So far, ESR has been the most widely used parameter to describe the inflammatory reaction in SLE. However, suPAR is a more stable and reliable parameter for the detection of inflammation than ESR, which might be influenced by several other factors (such as anemia). In further contrast with ESR, suPAR might be especially suitable for identifying patients with active disease, since suPAR values are elevated in patients with high disease activity in comparison with moderate disease activity lupus patients and those in remission. Hence, suPAR represents an objective clinical marker that can reliably be used for the detection of ongoing inflammation with respect to disease severity in SLE.

\section{Declaration of interest}

This work was supported by the Hungarian Scientific Research Fund [OTKA; grant number 101661], the Kerpel-Fronius Talent Support Program of Semmelweis University and an unrestricted research grant from
Roche Hungary Ltd. None of the authors has any conflict of interest related to this study.

\section{References}

Balabanov R, Lisak D, Beaumont T, Lisak RP, Dore-Duffy P. (2001). Expression of urokinase plasminogen activator receptor on monocytes from patients with relapsing-remitting multiple sclerosis: effect of glatiramer acetate (copolymer 1). Clin Diagn Lab Immunol 8:1196-1203.

Behrendt N, Stephens RW. (1998). The urokinase receptor. Fibrinolysis and Proteolysis 12:191-204.

Danø K, Behrendt N, Brünner N, Ellis V, Ploug M, Pyke C. (1994). The urokinase receptor. Protein structure and role in plasminogen activation and cancer invasion. Fibrinolysis 8:189-203.

de Carvalho JF, Hanaoka B, Szyper-Kravitz M, Shoenfeld Y. (2007). C-Reactive protein and its implications in systemic lupus erythematosus. Acta Reumatol Port 32:317-322.

Griffiths B, Mosca M, Gordon C. (2005). Assessment of patients with systemic lupus erythematosus and the use of lupus disease activity indices. Best Pract Res Clin Rheumatol 19:685-708.

Hochberg MC. (1997). Updating the American College of Rheumatology revised criteria for the classification of systemic lupus erythematosus. Arthritis Rheum 40:1725.

International Council for Standardization in Haematology (Expert Panel on Blood Rheology). (1993). ICSH recommendations for measurement of erythrocyte sedimentation rate. J Clin Pathol 46:198-203.

Liou LB. (2001). Serum and in vitro production of IL-1 receptor antagonist correlate with C-reactive protein levels in newly diagnosed, untreated lupus patients. Clin Exp Rheumatol 19:515-523.

Liou LB. (2003). Different monocyte reaction patterns in newly diagnosed, untreated rheumatoid arthritis and lupus patients probably confer disparate C-reactive protein levels. Clin Exp Rheumatol 21:437-444.

May AE, Neumann FJ, Schömig A, Preissner KT. (2000). VLA-4 (alpha(4) beta(1)) engagement defines a novel activation pathway for beta(2) integrin-dependent leukocyte adhesion involving the urokinase receptor. Blood 96:506-513.

Ostergaard C, Benfield T, Lundgren JD, Eugen-Olsen J. (2004). Soluble urokinase receptor is elevated in cerebrospinal fluid from patients with purulent meningitis and is associated with fatal outcome. Scand J Infect Dis 36:14-19. 
Ostrowski SR, Ullum H, Goka BQ, Høyer-Hansen G, Obeng-Adjei G, Pedersen BK, Akanmori BD, Kurtzhals JA. (2005). Plasma concentrations of soluble urokinase-type plasminogen activator receptor are increased in patients with malaria and are associated with a poor clinical or a fatal outcome. J Infect Dis 191:1331-1341.

Riisbro R, Christensen IJ, Høgdall C, Brünner N, Høgdall E. (2001). Soluble urokinase plasminogen activator receptor measurements: influence of sample handling. Int J Biol Markers 16:233-239.

Rønne E, Pappot H, Grøndahl-Hansen J, Høyer-Hansen G, Plesner T, Hansen NE, Danø K. (1995). The receptor for urokinase plasminogen activator is present in plasma from healthy donors and elevated in patients with paroxysmal nocturnal haemoglobinuria. Br J Haematol 89:576-581.

Russell AI, Cunninghame Graham DS, Shepherd C, Roberton CA, Whittaker J, Meeks J, Powell RJ, Isenberg DA, Walport MJ, Vyse TJ. (2004). Polymorphism at the C-reactive protein locus influences gene expression and predisposes to systemic lupus erythematosus. Hum Mol Genet 13:137-147.

Sier CF, Stephens R, Bizik J, Mariani A, Bassan M, Pedersen N, Frigerio L, Ferrari A, Danø K, Brünner N, Blasi F. (1998). The level of urokinase-type plasminogen activator receptor is increased in serum of ovarian cancer patients. Cancer Res 58:1843-1849.

Sier CF, Sidenius N, Mariani A, Aletti G, Agape V, Ferrari A, Casetta G, Stephens RW, Brünner N, Blasi F. (1999). Presence of urokinasetype plasminogen activator receptor in urine of cancer patients and its possible clinical relevance. Lab Invest 79:717-722.
Sjöwall C, Eriksson P, Almer S, Skogh T. (2002). Autoantibodies to C-reactive protein is a common finding in SLE, but not in primary Sjögren's syndrome, rheumatoid arthritis or inflammatory bowel disease. J Autoimmun 19:155-160.

Stephens RW, Pedersen AN, Nielsen HJ, Hamers MJ, Høyer-Hansen G, Rønne E, Dybkjaer E, Danø K, Brünner N. (1997). ELISA determination of soluble urokinase receptor in blood from healthy donors and cancer patients. Clin Chem 43:1868-1876.

Kravitz MS, Shoenfeld Y. (2006). Autoimmunity to protective molecules: is it the perpetuum mobile (vicious cycle) of autoimmune rheumatic diseases? Nat Clin Pract Rheumatol 2:481-490.

Tarui T, Mazar AP, Cines DB, Takada Y. (2001). Urokinase-type plasminogen activator receptor (CD87) is a ligand for integrins and mediates cell-cell interaction. J Biol Chem 276:3983-3990.

ter Borg EJ, Horst G, Limburg PC, van Rijswijk MH, Kallenberg CG. (1990). C-reactive protein levels during disease exacerbations and infections in systemic lupus erythematosus: a prospective longitudinal study. J Rheumatol 17:1642-1648.

Toldi G, Bíró E, Szalay B, Stenczer B, Molvarec A, Rigó J, Vásárhelyi B, Bekõ G. (2011). Soluble urokinase plasminogen activator receptor (suPAR) levels in healthy pregnancy and preeclampsia. Clin Chem Lab Med 49:1873-1876.

Toldi G, Bekó G, Kádár G, Mácsai E, Kovács L, Vásárhelyi B, Balog A. (2012). Soluble urokinase plasminogen activator receptor (suPAR) in the assessment of inflammatory activity of rheumatoid arthritis patients in remission. Clin Chem Lab Med 0:1-6. 